Aus der kaiserlichen dermato-urologischen Universitätsklinik in Tokso, Japan. (Direktor: Professor Dr. K. Dohi.)

\title{
Ein Beitrag zur Pathologie und Statistik der Epididymitis gonorrhoica.
}

Von

Dr. T. Tanaka aus Tokyo, Japan.

Die Epididymitis gonorrhoica, die als eine Komplikation der Urethritis genorrhoica des Mannes auftritt, treffen wir unter allen Komplikationen nächst der Cystitis cerbicale am häufigsten an.

Da die Epididymitis oft die Zeugungsfunktion beschädigt und also die Fruchtlosigkeit verursacht, so hat diese Krankheit unter allen Tripperkomplikationen das innigste Verhältnis zur Sozialfrage und verdient in dieser Hinsicht unsere genaue Untersuchung auf die Verhältniszabl ihres Auftretens hin. Leider haben wir noch keine zuverlässige statistischen Arbeiten über diese Krankheit, und verschiedene Berichte verschiedener Autoren hierüber zeigen immer noch verschiedene Ziffern. Schäffer (1) meint, daß der Tripper bei dem Manne sehr häufig vorkommt, der in Folge dessen an der Nebenhodenentzündung auch häufig leidet und daher dem Arzt keine genaue richtige Klage über seine Krankheit macht.

Außerdem hält Finger (2) die Statistik über die Häufigkeit der Epididymitis nicht für genau, die dem Hospitalmaterial entnommen sind, da die Patienten aber erst dann das Hospital aufzusuchen pflegen, wenn sie schon lange an der Nebenhodenentzündung gelitten haben und wegen der Schmerzen nicht mehr arbeiten können. Aber auch die ambulatorisch behandelten Tripperkranken können nicht ohne weiteres ein 
richtiges Bild liefern, da sie oft die Heilung unterlassen, sich an einen anderen Arzt wenden, oder von ihrer Anamnese keine richtige Angabe machen. So müssen wir die Statistik über das Hospitalmaterial von dem über die ambulatorisch behandelten Kranken genau unterscheiden. Falls aber ein Patient einmal ambulatorisch behandelt und dann ins Hospital aufgenommen worden ist, so ist eine solche Unterscheidung nicht mehr nötig. Immerhin müssen wir den Fall ins Auge fassen, daß ein und derselbe Kranke mehrere Male am Tripper leidet, oder schon lange vom Tripper überfallen und an der chronischen Erkrankung leidet und infolge von Unmäßigkeit und schlechter Behandlung manchmal Exacerbationen erleidet und jedesmal die Nebenhodenentzündung wieder weckt. Ferner muß man berücksichtigen, daß es solche Tripperkranke gibt, welche ihrer leichten Erkrankung und stumpfer Ëmpfiıdung wegen von ihrer Krankheit gar keine Ahnung haben und selbst bei der Eintretung kleiner Schmerzen diese für eine Art Neuralgie halten. Einige Kranke sind ja noch stumpfsinniger, daß sie verhältnismäßig ernstere Schmerzen und sogar gewisse Erhitzung einer infektiösen Erkältung zuschreiben und erst bei der Nebenhodenanschwellung auf die richtige Meinung kommen, daß sie von der Nebenhodenentzündung überfallen worden sind. Aus dem gesagten ergibt sich, daß die bloß temporal verlaufende Entzündung weder vom Kranken noch dem Arzt verspürt werden kann und somit unserer statistischen Betrachtung entgehen muß. Außerdem ist hier erwühnenswert, daß die Urethritis nongonorrhoica resp. die Urethritis gonorrhoica eine Mischinfektion verursacht und indem durch die Bakterien eine Epididymitis nongonorrhoica zu stande kommt. Darum sah Bockhart (3) in einer Urethritis, die von den aus der Geschlechtshöhle infektiös fortgepflanzten Spaltepilzen, Mikrokokken und Streptokokken oroida erweckt wurde, wohl die Ursache der Nebenhodenentzündung. Gusta v (4) sah auch faktisch, wie die Urethritis nongonorrhoica die Nebenhodenentzïndung erzeugt. Bernstein (5) besagt, daß Friedländersche Bakterien ebenfalls eine Nebenhodenentzündung aufkommen lassen. Nach Galewslsy (6) erweckt die chronische Urethritis nongonorrhoica eine Neben- 
hodenentzündung, v. Hof fmann (7) erstattet uns eine wichtige Mitteilung über den Bakterienzustand in der Urethritis chronica, Elsenberg (8) über die Epididymitis idiopatbica und Naught (11) über die Epididymitis thyphosa. Außerdem treffen wir gar oft die tuberkulöse Epididymitis. So ist es unverkennbar nötig, alle solche Arten klar zu unterscheiden. Da aber diese Sache außerordentlich schwierig ist, so müssen wir unsere $\mathrm{Be}$ obachtung auf das Gebiet der klinisch als solche diagnostizierten Epididymitis gonorrhoica beschränken. Zunächst folge die Tabelle, welche die von verschiedenen Autoren diesbezüglich mitgeteilten Ziffern ersichtlich macht.

Tabelle I.

\begin{tabular}{|c|c|c|c|c|c|c|}
\hline A $u t \circ r$ & 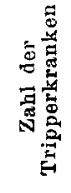 & 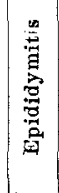 & 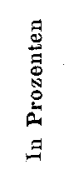 & 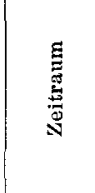 & 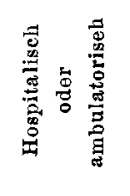 & $\begin{array}{c}O \mathrm{r} \mathrm{t} \\
\text { der Behandlung }\end{array}$ \\
\hline Baraczynski (9) & - & -- & $3 \cdot 2$ & - & Hospitäler & Wien \\
\hline Finger $(2) . .$. & 1844 & 548 & $29 \cdot 9$ & 5J. lang & " & Leipzig u. Wien \\
\hline Weber $(9)$. & - & - & $28 \cdot 2$ & 1890 & $n$ & Würzburg \\
\hline Rollert (2). . . & 2425 & 678 & $27 \cdot 9$ & - & - & - \\
\hline Simonis, J. (10) . & 一 & $一$ & $\therefore 7 \cdot 5$ & $1897-$ & 一 & - \\
\hline $\left.\begin{array}{l}\text { Tichomirow (9) } \cdot \\
\text { Pusenkin }(9) \cdot . \\
\text { Smirnow (9). }\end{array}\right\}$ & 2386 & 406 & $17 \cdot 3$ & $\begin{array}{l}1901 \\
1886\end{array}$ & ambulator. & Moskau \\
\hline Jullien (2) . . . . & 2500 & 381 & $15 \cdot 2$ & 1903 & $"$ & Leipzigu. Wien \\
\hline Pezzoliu.Porges $(9)$ & 3934 & 560 & $14 \cdot 2$ & 1872 & $"$ & Petersburg \\
\hline Tarnowsky (2) . . & 5203 & 637 & $12 \cdot 2$ & - & $n$ & - \\
\hline Finger $(2) \cdot . \cdot$ & 1000 & 125 & $12 \cdot 5$ & 1901 & $n$ & - \\
\hline Le Clere,Daudry $(9)$ & - & - & $12 \cdot 0$ & 1900 & $\eta$ & Moskau \\
\hline Tarnowsky (9) . . & 657 & 77 & $11 \cdot 7$ & 1904 & " & $n$ \\
\hline Jordan $(9) \ldots$. & 812 & 91 & $11 \cdot 7$ & - & - & - \\
\hline Gassmann (12). . & 44 & 5 & $11 \cdot 3$ & 1904 & $"$ & Bern \\
\hline Wagapow (9) . & - & 103 & $8 \cdot 4$ & 1896 & - & - \\
\hline Berg (2) . . . & - & - & 7.5 & 1882 & 一 & - \\
\hline Gebert $(9)$. . . & 650 & 一 & $7 \cdot 0$ & 1893 & - & - \\
\hline
\end{tabular}


v. Z e is si (13) gibt in seinem Lehrbuch die Differenz von $10-30 \%$ und $S \mathrm{ch}$ äffer die von $4-40 \%$ an.

Die Angabe von Patienten ist nicht immer richtig. Es ist z. B. sehr möglich, daß einer die Hodenanschwellung für die Nebenhodenentzündung halten kann und auf diese Weise dem Arzt eine falsche Mitteilung macht. So muß der Arzt immer vorsichtig sein und auf den Zustand des betreffenden Patienten möglichst eingehende Untersuchung machen.

Es wird viel gestritten, ob die Epididymitis gonorrhoica mehr die linke Seite angreift oder die rechte. In Europa ist die Meinung entstanden, daß diese Krankheit meistens die linke Seite überfällt, da man den Geschlechtsteil an der linken Seite der Hosen trägt, und die linke Hode mehr Druck und Zug erleidet als die rechte. Finger (2) behauptet dagegen, daß diese Unterscheidung gar nicht nötig ist, da diese Differenz bei der vielseitigen Beleuchtung wenig von Bedeutung ist. $\mathrm{Z} e$ issl aber sagt, daß die Krankbeit mehr auf der linken Seite auftritt. Außerdem gibt es verschiedene Ansichten, die bald die linke, bald die rechte Seite als die häufiger erkrankte hinstellen. Immerhin ist die Differenz stets sehr klein. Jedenfalls ist die Erkrankung der beiden Seiten sehr selten und die gleichzeitige Erkrankung der beiden Seiten noch weit seltener. Gewöhnlich beginnt sie von irgend einer Seite, $d . h$. von links oder rechts. Nun die hier bezüglichen Ziffern verschiedener Autoren:

Tabelle II.

\begin{tabular}{|c|c|c|c|c|c|c|c|}
\hline \multirow[b]{2}{*}{$A$ u $t$ or } & \multicolumn{2}{|c|}{ Link $\theta$ Seite } & \multicolumn{2}{|c|}{ Rechte Seite } & \multicolumn{2}{|c|}{ Boide Seiten } & \multirow{2}{*}{ 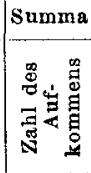 } \\
\hline & 总密 & 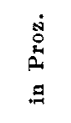 & 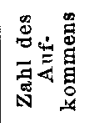 & 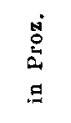 & 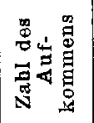 & $\begin{array}{l}\text { D. } \\
\stackrel{0}{0} \\
\text { R } \\
\Xi\end{array}$ & \\
\hline 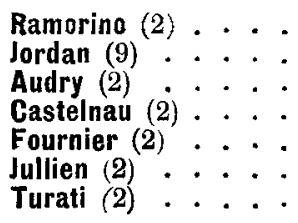 & $\begin{array}{r}37 \\
49 \\
52 \\
133 \\
126 \\
182 \\
192\end{array}$ & $\begin{array}{l}56 \cdot 1 \\
53 \cdot 8 \\
52 \cdot 0 \\
50 \cdot 1 \\
47 \cdot 9 \\
47 \cdot 6 \\
44 \cdot 1\end{array}$ & $\begin{array}{r}29 \\
32 \\
40 \\
125 \\
102 \\
167 \\
191\end{array}$ & $\begin{array}{l}43 \cdot 9 \\
35 \cdot 1 \\
39 \cdot 4 \\
47 \cdot 2 \\
41 \cdot 8 \\
43 \cdot 7 \\
46 \cdot 8\end{array}$ & $\begin{array}{r}0 \\
10 \\
7 \\
7 \\
35 \\
33 \\
25\end{array}$ & $\begin{aligned} 0 \\
11 \cdot 1 \\
8 \cdot 6 \\
2 \cdot 7 \\
10 \cdot 3 \\
8 \cdot 7 \\
6 \cdot 1\end{aligned}$ & $\begin{array}{r}66 \\
91 \\
99 \\
265 \\
263 \\
382 \\
408\end{array}$ \\
\hline Fürtrag & 771 & - & 686 & - & 117 & - & 1574 \\
\hline
\end{tabular}


Ein Beitrag zur Pathol. und Statistik der Epididymitis gon. 239

\begin{tabular}{|c|c|c|c|c|c|c|c|}
\hline \multirow[b]{2}{*}{$A$ ut $0 \mathrm{r}$} & \multicolumn{2}{|c|}{ Linke Seito } & \multicolumn{2}{|c|}{ Rechte Seite } & \multicolumn{2}{|c|}{ Beide Seiten } & \multirow{2}{*}{ 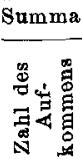 } \\
\hline & 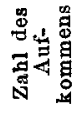 & 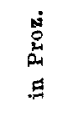 & 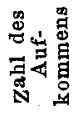 & 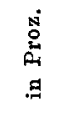 & 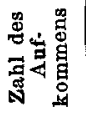 & 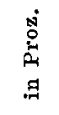 & \\
\hline Übertrag . . & 771 & - & 686 & - & 117 & - & 1574 \\
\hline \multirow{12}{*}{ 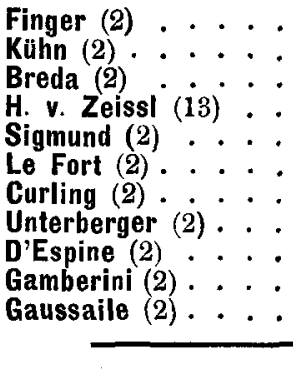 } & 251 & $45 \cdot 8$ & 275 & $50 \cdot 1$ & 22 & $4 \cdot 1$ & 548 \\
\hline & 67 & $44 \cdot 9$ & 70 & 46.9 & 12 & $8 \cdot 2$ & 149 \\
\hline & 53 & $43 \cdot 8$ & 64 & $54 \cdot 8$ & 4 & $1 \cdot 4$ & 121 \\
\hline & 33 & $43 \cdot 4$ & 36 & $47 \cdot 3$ & 7 & $10 \cdot 3$ & 76 \\
\hline & 48 & $42 \cdot \overline{1}$ & 60 & $52 \cdot 6$ & 6 & $5 \cdot 3$ & 114 \\
\hline & 200 & $40 \cdot 8$ & 249 & $50 \cdot 8$ & 41 & $8 \cdot 4$ & 490 \\
\hline & 14 & $38 \cdot 8$ & 21 & $58 \cdot 3$ & 1 & $2 \cdot 9$ & 36 \\
\hline & 25 & $38 \cdot 4$ & 35 & $53 \cdot 8$ & 5 & $7 \cdot 8$ & 65 \\
\hline & 11 & 37 . & 12 & $41 \cdot 4$ & 6 & $20 \cdot 7$ & 29 \\
\hline & 10 & $35 \cdot 7$ & 15 & $53 \cdot 5$ & $\mathbf{3}$ & $10 \cdot 8$ & 28 \\
\hline & 24 & $32 \cdot 9$ & 45 & $61 \cdot 6$ & 4 & $5 \cdot 5$ & 73 \\
\hline & 1507 & $45 \cdot 6$ & 1568 & $47 \cdot 4$ & 228 & $6 \cdot 9$ & 3303 \\
\hline
\end{tabular}

Tabelle III.

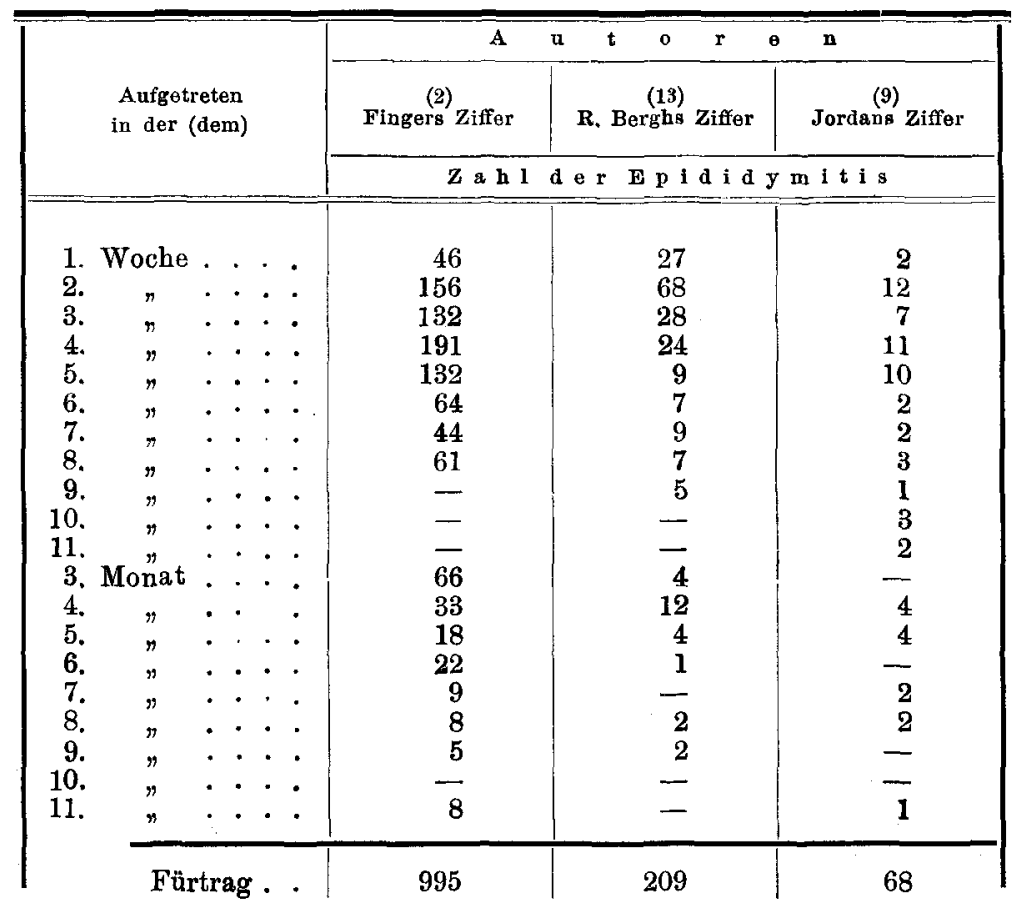




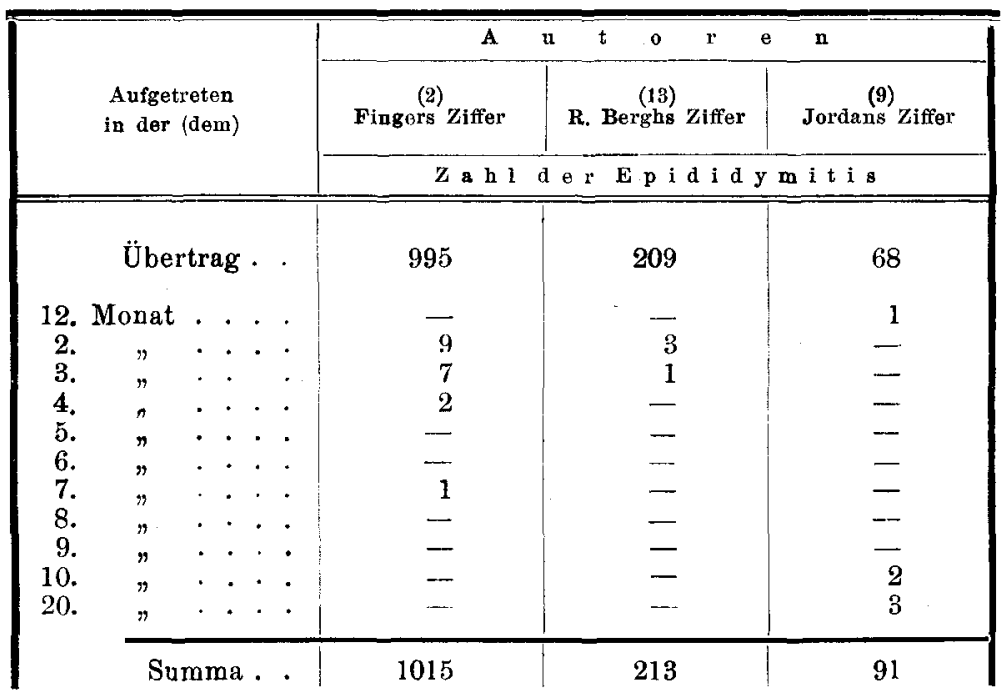

Das Maximum der Nebenhodenentzündungen durch die Urethritis gonorrhoica fällt auf das Ende der 3. Woche oder den Anfang der 4. Woche des Trippers, wo die Urethritis anterior auf die Urethritis posterior übergeht, d. h. das akute Stadium auf das subakute. Falls man im Anfange des akuten Stadiums mittels eines Instrumentes den Eiter in die innere Tiefe hineintreibt und somit eine Urethritis posterior zu Stande bringt, so kann man in einer Woche schon die Nebenhodenentzündung wahrnehmen. Wie die folgenden Statistiken von Finger, Bergh und Jordan (9) uns zeigen, tritt die Nebenhodenentzündung innerhalb der 5. Woche nach der Infektion des Trippers fast ebenso oft auf, wie die Krankheit selbst wegen der Steigerung der Erkrankung im Verlaufe des chronischen Stadiums.

Zahl und Zeit des Auftretens der Epididymitis nach der Dauer des Trippers siehe in der vorstehenden Tabelle III.

Was die Jahreszeiten anbelangt, so ist früher einmal von Fing e r (14) auf Grund von Beobachtungen an der Z e is s lschen Klinik die Vermutung ausgesprochen worden, daß die Jahreszeiten für die Entstehung der Nebenhodenentzündung Bedeutung haben, insofern, als das Maximum derselben in 
die Zeit der anhaltenden Trockenheit und größten Hitze fallen soll. Dementgegen spricht sich Jor $\mathrm{d}$ a $\mathrm{n}$ aus, daß die Jahreszeiten keinen namhaften Einfluß darauf hatten.

Auch das Alter der Tripperkranken hat nach Jordan keinen besonderen Einfluß auf das Auftreten der Epididymitis. Er hat bloß anerkannt, daß in der Periode, wo Trippererkrankungen zahlreich sind, die Nebenhodenentzündungen gleichfalls zahlreich sind.

Über die Verhältniszahl der Nebenhodenentzündungen und deren Behandlungen haben wir Le Forts Bericht. Der ist folgender:

Art und Weise der Behandlung und Pflege:

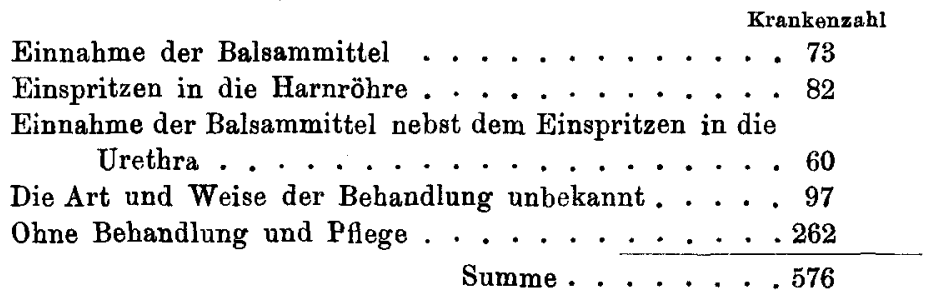

Das Auftreten der Nebenhodenentzündung ist, um mit Finger zu reden, bei unbehandelten Tripperkranken am häufigsten. Diejenigen Patienten, die die Krankheitspflege vernachlässigen, müssen ebenfalls oft an der Nebenhodenentzündung leiden. Doch ist diese Krankheit nicht der vernachlässigten Behandlung allein zuzuschreiben.

Die vorliegende Arbeit ist im Zeitraum von rund 2 Jahren (Januar 1903 bis Dezember 1904) auf dem Ambulatorium des Professor Dr. K. Dohi an der dermatologischen Abteilung in der medizinischen Fakultät zu Tokyo unternommen worden. Die Gesamtzahl der hier ambulatorisch behandelten Patienten betrug 6218, worunter 4602 Männer waren. Der jüngste dieser Tripperkranken war 17 Jahre alt. Wir hatten also 4045 Männer, die alle über 17 Jahre alt waren und in unseren Betrachtungskreis gezogen werden sollten. Die Tripperkranken betrugen 674, und die Prozentzahl war also $16 \cdot 7 \%$. Zählen wir die Männer über 17 und unter 40 Jahre alt, so ist ihre Gesamtzahl 3695 , d. h. $18 \cdot 2 \%$. Jedenfalls ist meine 
Zahl kleiner als die des Okamura (15). Diese Differenz läßt sich aber dadurch erklären, daß ich bloß die gegenwärtig leidenden Kranken zählte, während $\mathrm{Okamura}$ auch die Fälle in der Anamnese mitzählte.

Jordan zählt unter 493 dermatologischen und urologischen Kranken, über 19 Jahre alt, 48\% Tripperkranke. Erb (16) sah unter über 25jährigen Kranken 50\% Tripperkranke. Die Verhältniszahlen der beiden Autoren sind wohl größer als die meinigen. In der Tat baben wir aber neuerdings durchschnittlich 883 zweimalige Patienten gehabt, unter denen 728 Männer sind, und von ihnen leiden 314 an Tripper, die übrigen aber an Hautleiden oder Syphilis. So bietet sich uns die Verhältniszahl der Tripperkranken als $43 \cdot 1 \%$ dar, was Jordans Ziffer näher steht.

1. Verhältnis der Nebenhodenentzündung zur Urethritis.

Unter meinen 674 Tripperkranken fanden sich 75 Patienten, welche zur Zeit der Untersuchung gerade an der Nebenhodenentzündung leidend waren; es macht also $11 \cdot 1 \%$.

Unter der Nebenhodenentzündung habe ich auch diejenige Krankheit verstanden, welche die Peridiferentitis resp. Funiculitis begleitet und auf dem betreffenden Teile des Leidens eine starke Infiltration verursacht, ferner diejenige Krankheit, welche am Nebenhoden zwar keine Infiltration verspüren läßt, doch gewissermaßen Schmerzen erweckt. Denn solche Zustände sind pathologisch sowohl wie histologisch schon als Kanälchenentzündungen anzusehen. Die Erkrankung, die außer der Verdickung und Verhärtung des Nebenhoden auch die Verdickung der Spermatis erzeugt, habe ich natürlich hier mitgezählt. In meinen bisherigen Untersuchungen sah ich noch keine Spermatisentzündung, welche mit Störung am Nebenhoden nicht in Gesellschaft auftrat.

Was aber die leichten Entzündungen in Begleitung mit der vorübergehenden Ejaculatoritis, Deferentitis und Kanälchenentzündung ohne besonderen Schmerz und ohne Spur der 
Ein Beitrag zur Pathol. und Statistik der Epididymitis gon. 243

Infiltration anbelangt, so können wir noch nicht auf eine Nebenhodenentzündung schließen. Wenn aber die Erkrankung Hitze erweckt oder gewisse drückende Lokalschmerzen verursacht, dann können wir sie zu unserer Statistik gebrauchen. Erweckt sie dagegen bloß einen leichten Schmerz, so können wir sie nicht gleich zu unserem Gebiete mitzählen, da ein solcher Schmerz ebenso gut auch von der Neuralgie herkommen kann. Falls aber die Körpertemperatur steigt und Lokalschmerzen heftig werden, dann ist es klar, daß die Nebenhodenentzündung schon eingetreten ist, und man kann dann sogar eine gewisse Verdickung wahrnehmen. Von der Anamnese des Patienten kann man keine Grenauigkeit erwarten, da die Patienten nach dem Verlaufe vieler Jahre den größeren Teil ihrer vergangenen Erkrankungen vergessen haben. Unter meinen Patienten gab es 18, die sich an ihre damaligen Nebenhodenentzündungen noch erinnern konnten. Die Gesamtzahl meiner Epididymitiskranken betrug 93, einschließlich der gegenwärtig leidenden Kranken, die Verhältniszahl ist demmach $13.8 \%$ (s. IV. Tabelle). Diese Ziffer kommt noch an Gassmann (12) $11.3 \%$, Jordans $11.7 \%$ und Tarnowskys $11 \cdot 7 \%$. Diese Ziffern beziehen sich alle auf die Erkrankungen der ambulatorisch Behandelten. Die Art und Weise ihrer Untersuchung deckt die meinige. Jordan teilt uns unter 812 Patienten 135 Epididymitisfälle mit, einschließlich der Erkrankungen in der Anamnese, d. i. also $27.8 \%$ (diese statistische Arbeit ist in Moskau unternommen). Da die Japaner aber stets mit einem ziemlich breiten Band die Hoden befestigen, so soll bei ihnen die äußerliche Trauma dieses Teils verhältnismäßig seltener sein als bei denen, welche ihre Hoden nur frei herabhängen lassen.

\section{Die Seite.}

Unter meinen 93 Patienten bekamen 54 am linken Hoden Entzündung, das ist $58 \cdot 1 \%, 32$ aber am rechten Hoden, d. i. $34.4 \%$ und 7 endlich auf den beiden Seiten, d. i. $7 \%$. So stehen meine Ziffern nahe an den Prozentzahlen Jordans 
und Ramorinos, sind aber ron denen Curlings und Unt erbergers entfernt. Selbst die beiderseitige Erkrankung beginnt gewöhnlich zunächst von der rechten Seite. Die Frist des Übergangs der Entzündung von einer Seite zur anderen dauert fast eine Woche. Bei meinen 7 Fällen dehnte sich diese Frist 3-10 Tage aus, ausgenommen eine dreimonatliche und eine halbjährige. Eine gleichzeitig auf den beiden Seiten aufkommende Entzündung habe ich noch nicht angetroffen.

Tabelle IV.

\begin{tabular}{|c|c|c|c|c|c|c|c|c|c|}
\hline \multirow{3}{*}{$\mathrm{z}$ e i t $\mathrm{raum}$} & \multicolumn{4}{|c|}{$\begin{array}{l}\text { Krankhait } \\
\text { bei der Anamnese }\end{array}$} & \multicolumn{4}{|c|}{$\begin{array}{l}\text { Krankheit } \\
\text { bei der Gegenwrart }\end{array}$} & \multirow{3}{*}{ 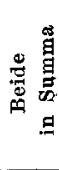 } \\
\hline & 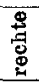 & $\stackrel{\text { I }}{\text { I }}$ & 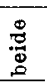 & \multirow{2}{*}{ 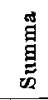 } & 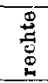 & 苞 & 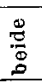 & \multirow{2}{*}{$\begin{array}{l}\text { 韋 } \\
\text { 昜 }\end{array}$} & \\
\hline & \multicolumn{3}{|c|}{ Seite } & & \multicolumn{3}{|c|}{ Seite } & & \\
\hline $1903 . . . . . \cdot$ & 2 & 3 & 1 & 6 & 12 & 19 & 2 & 33 & 39 \\
\hline $1904 . . . . . \cdot$ & 5 & 6 & 1 & 12 & 13 & 26 & 3 & 42 & 54 \\
\hline $1903-04 \ldots$ & 7 & 9 & 2 & 18 & 25 & 45 & 5 & 75 & 93 \\
\hline
\end{tabular}

3. Welches Stadium der Urethritis gonorrhoica bringt die Nebenhodenentzündung zu Stande?

Am häufigsten kommt die Nebenhodenentzündung, wie die Tabellen Fingers (s. o.), R. Berghs, sowohl wie J ordan es zeigen, in der Zeit zustande, wo ein Tripper vom akuten Stadium über das subakute zum chronischen übergeht, $d . h$. in der Periode, wo eine Urethritis posterior aufgekommen ist, oder wo sich die Erkrankung in chronischem Verlaufe wegen der Unmäßigkeit des Patienten oder des schlechten Klimas besonders heftig gestaltet. Fing e $r$ behauptet sogar, daß auch bei akutem Verlaufe eine Nebenhodenentzündung zu Stande gebracht werden kann, falls pathogene Bakterien vermittelst einer Bougierung oder Einspritzung in den hinteren Teil hineingetrieben werden. Nach meiner Tabelle (V) ist die betreffende Erkrankung während des Zeitraums 6-28 Tage nach dem Aufkommen der Urethritis am häufigsten, aber je später desto seltener. 
Tabelle V.

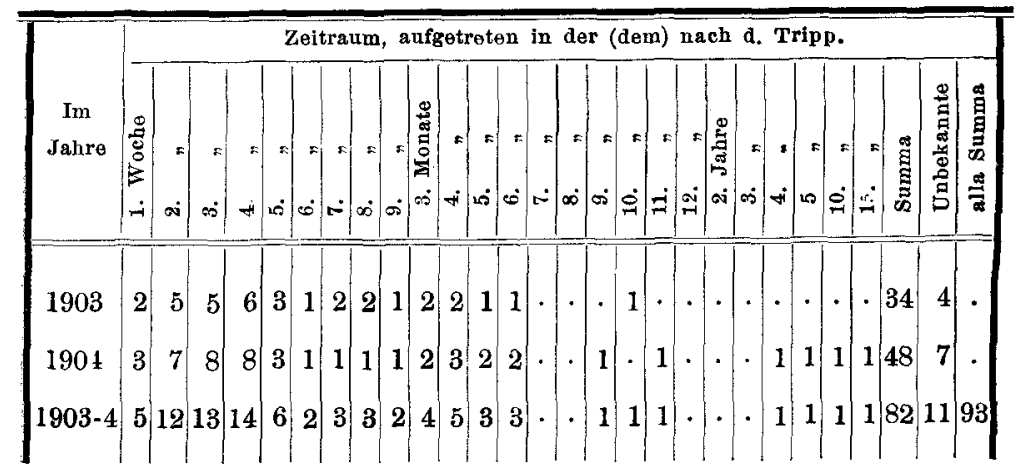

4. Die Jahreszeiten.

Untersuchen wir, in welcher Jahreszeit die Nebenhodenentzündung am häufigsten vorkommt, so scheint es sehr wahrscheinlich, dab die Periode ihrer Häufigkeit mit der des Tripperanfalles identisch sei. Doch läßt es sich nicht so leicht bestimmen. Nach meiner VI. Tabelle steigert sich die Prozentzahl in der Zeit, wo das Klima sich ändert. In der Periode, wo die Kälte zur Wärme und die Wärme zur Kälte übergeht, also im März, Juni und November wird die Prozentzahl größer im Vergleich zu anderen Monaten. Denn in der Zeit der Klimaveränderung erleidet jeder Organismus gewisse Veränderung, und dem Menschen wird es in dieser Zeit auch schwierig, seine Gesundheit gut zu halten. Die Feuchtigkeit, Temperatur sowohl wie die Dichte der Luft bis auf die Veränderung des Trinkwassers bleiben nicht ohne namhaften Einfluß auf die Gesundheit des Menschen. Übrigens kann es auch der Fall sein, daß im Frühling und Herbste die sinnliche Leidenschaft der Menschen heftiger wird und viele Menschen gerade in dieser Zeit durch Fehltritte die Nebenhodenentzündung auf sich laden. Finger sagte, daß die Nebenhodenentzündung zur Zeit der großen Trockenheit und Hitze am häufigsten vorkommt. Hiermit wollte er sagen, daß die Häufigkeit des Auftretens dieser Krankheit auf die Zeit der größten Kälte und Wärme fällt. Die Behauptung 
aber entspricht nicht meinen Fällen. Man achte aber in der Tabelle darauf, daß die verhältnismäßig große Häufigkeit der Nebenhodenentzündung im März, Juni nnd November nicht immer von der Häufigkeit des akuten resp. subakuten Trippers abhängig ist.

Tabelle VI.

Urethritis gonorrhoica des Mannes.

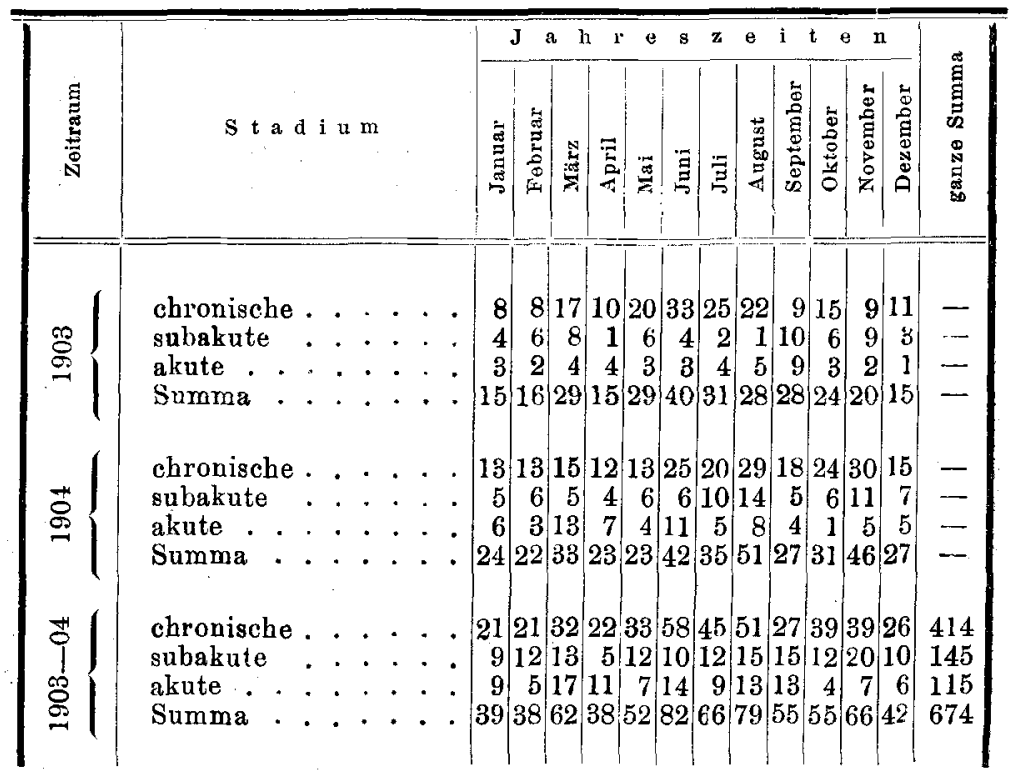

Epididy mitis gonorrhoica.

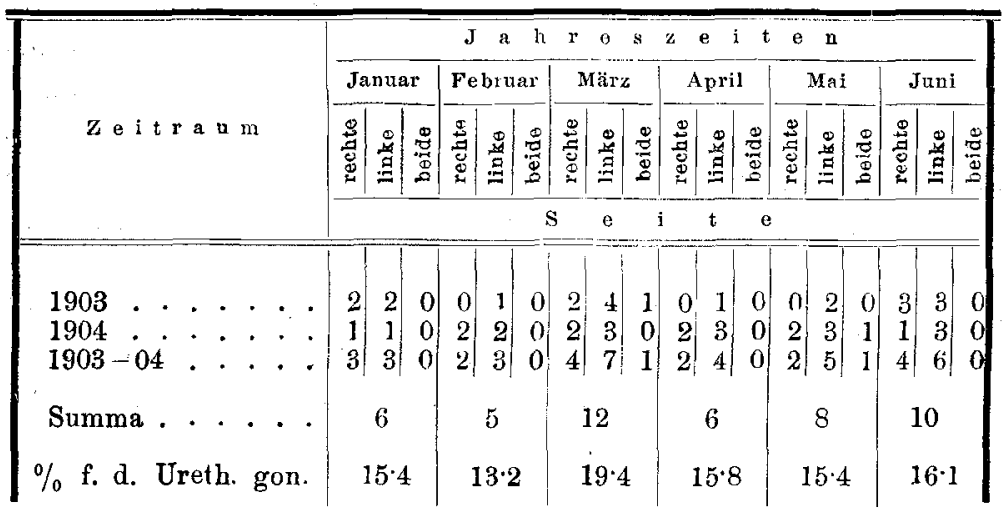


Ein Beitrag zur Pathol. und Statistik der Epididymitis gon. 247

\begin{tabular}{|c|c|c|c|c|c|c|c|c|c|c|c|c|c|c|c|}
\hline \multirow[b]{3}{*}{ Zoitra um } & \multicolumn{14}{|c|}{ 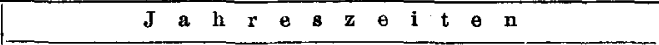 } & \multirow{3}{*}{ 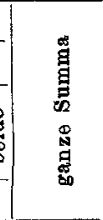 } \\
\hline & \multicolumn{2}{|c|}{ Juli } & \multicolumn{2}{|c|}{ August } & \multicolumn{3}{|c|}{ Septbr. } & \multicolumn{3}{|c|}{ Oktober } & \multicolumn{2}{|c|}{ Novbr. } & \multicolumn{2}{|c|}{ Dezbr. } & \\
\hline & 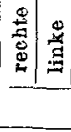 & $\mid$ & & : & \pm & $\frac{0}{0}$ & $\stackrel{0}{0}$ & & $\frac{\stackrel{D}{B}}{t}$ & $\frac{0}{0}$ & 量 & $\mid$ & 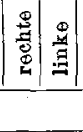 & : & \\
\hline $\begin{array}{l}1903 . \\
1904: 04 \\
1903-04:\end{array}$ & \begin{tabular}{l|l}
1 & 0 \\
1 & 5 \\
2 & 5
\end{tabular} & $\mid \begin{array}{l}1 \\
1 \\
2\end{array}$ & \begin{tabular}{l|l}
1 & 5 \\
2 & 5 \\
3 & 7
\end{tabular} & $\begin{array}{l}1 \\
1 \\
1 \\
2\end{array}$ & & $\left|\begin{array}{l}2 \\
2 \\
4\end{array}\right|$ & $\begin{array}{l}0 \\
0 \\
0\end{array}$ & & $\begin{array}{l}1 \\
0 \\
1\end{array}$ & & \begin{tabular}{l|l|}
2 & 2 \\
3 & 4 \\
5 & 6
\end{tabular} & $\begin{array}{l}0 \\
0 \\
0\end{array}$ & \begin{tabular}{l|l}
1 & 1 \\
0 & 2 \\
1 & 3
\end{tabular} & & \begin{tabular}{l|l|l|}
14 & 22 & 3 \\
18 & 32 & 4 \\
32 & 54 & 7
\end{tabular} \\
\hline Summa . . & 9 & & 12 & & & 6 & & & 4 & & 11 & & 4 & & 93 \\
\hline $\begin{array}{l}\% \text { für die } \\
\text { Urethr. gon. }\end{array}$ & $13 \cdot$ & & 15 & & & $11 \cdot 0$ & & & $7 \cdot 3$ & & $16 \cdot 7$ & & $9 \cdot 5$ & & $13 \cdot 8$ \\
\hline
\end{tabular}

\section{Das Alter:}

Nach Jordan ist das Alter von keinem nennenswerten Einfluß auf die Nebenhodenentzündung. Dieser Meinung stimme ich vollständig bei. Wie es die IV. Tabelle zeigt, ist diese Krankheit am häufigsten in der Zeit, wo der Tripper am häufigsten ist, d. h. im kräftigen Mannesalter. Aber was die Häufigkeitszahl der Nebenhodenentzündung anbelangt, so ist sie nicht von der des Trippers abhängig; sie verändert sich vielmehr nach der Komplikationszahl des akuten und subakuten Verlaufs. Warum bilden aber die Alter von 15 bis 20 Jahren hier eine Ausnahme? Hierüber möchte ich eine Vermutung aussprechen, daß man in diesem Alter vor dem Tripper große Furcht hat, und also die Heilung desselben ängstlich pflegt, und daß Leute unter 20 Jahren gewöhnlich noch keinen bestimmten Beruf haben, so daß sie sich nicht dazu gezwungen sehen, wegen der gegebenen Arbeit sich Gewalt anzutun (s. VII. Tabelle).

\section{Der Beruf.}

Daß die Häufigkeit der Nebenhodenentzündung als eine Folge des Trippers von dem Berufe des Menschen beeinflußt werden kann, ist wohl denkbar. T a r n ow sky soll neulich in Petersburg, Moskau und Paris erfahren haben, daß die Zahl der Epididymitis von der des Trippers nicht sehr ent- 
fernt ist, da arme Tripperkranke dort erst nach dem Zustandekommen der Komplikationen das Hospital besuchen. Jor dan berichtet, die Epididymitis wird vermißt bei einem solchen Berufe, welcher wahrscheinlich eine große Häufigkeit dieser Krankheit verheißt. Auch bei solchen Berufen, welche Springen, Emporrichten, Ausschlagen usw. verlangen, ist die Epididymitis nicht zahlreicher.

Tabelle VII.

Urethritis gonorrhoica des Mannes.

\begin{tabular}{|c|c|c|c|c|c|c|c|c|c|c|c|}
\hline \multirow[b]{2}{*}{ 礐 } & \multirow[b]{2}{*}{ Stadium } & \multicolumn{9}{|c|}{ Alterin Jahren } & \multirow[b]{2}{*}{ Summa } \\
\hline & & वิ & $\frac{1}{a}$ & 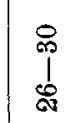 & $\frac{1}{\infty}$ & $\prod_{\infty}^{7}$ & $\frac{10}{7}$ & 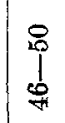 & $\frac{1}{20}$ & $\begin{array}{l}0 \\
0 \\
5 \\
0 \\
0\end{array}$ & \\
\hline 1903 & $\begin{array}{l}\text { chronische } \\
\text { subakute. } \\
\text { akute. . } \\
\text { Summa. . }\end{array}$ & $\begin{array}{r}11 \\
7 \\
4 \\
22\end{array}$ & $\begin{array}{l}51 \\
17 \\
17 \\
85\end{array}$ & $\begin{array}{l}57 \\
25 \\
12 \\
94\end{array}$ & $\begin{array}{r}28 \\
8 \\
7 \\
43\end{array}$ & $\begin{array}{r}16 \\
1 \\
1 \\
18\end{array}$ & $\left\{\begin{array}{r}10 \\
0 \\
1 \\
11\end{array}\right.$ & $\begin{array}{l}5 \\
0 \\
0 \\
5\end{array}$ & $\begin{array}{l}0 \\
0 \\
0 \\
0\end{array}$ & $\mid \begin{array}{l}2 \\
0 \\
0 \\
2\end{array}$ & $\begin{array}{l}- \\
-\end{array}$ \\
\hline 1904 & $\begin{array}{l}\text { chronische } \\
\text { subakute } \\
\text { aknte. } \\
\text { Summa. }\end{array}$ & $\begin{array}{r}22 \\
9 \\
13 \\
44\end{array}$ & $\begin{array}{r}89 \\
35 \\
30 \\
154\end{array}$ & $\begin{array}{r}52 \\
26 \\
24 \\
102\end{array}$ & $\begin{array}{r}35 \\
9 \\
3 \\
47\end{array}$ & $\begin{array}{r}17 \\
5 \\
2 \\
24\end{array}$ & $\begin{array}{l}4 \\
2 \\
1 \\
7\end{array}$ & $\begin{array}{l}6 \\
0 \\
0 \\
6\end{array}$ & $\begin{array}{l}4 \\
0 \\
0 \\
4\end{array}$ & $\mid \begin{array}{l}5 \\
0 \\
0 \\
5\end{array}$ & $\begin{array}{l}- \\
-\end{array}$ \\
\hline $1903-4$ & $\begin{array}{l}\text { chronische } \\
\text { subaknte } \\
\text { akute.. } \\
\text { Summa. . }\end{array}$ & $\mid \begin{array}{l}33 \\
16 \% \\
178 \\
66\end{array}$ & $\mid \begin{array}{r}140 \\
529 \\
47 . \\
239\end{array}$ & $\mid \begin{array}{l}109 \\
510 \\
36 \\
196\end{array}$ & $\begin{array}{l}630 \\
17 \stackrel{\circ}{10} \\
10 \dot{9} \\
90^{\circ}\end{array}$ & $\begin{array}{c}33 \\
6 \stackrel{\circ}{\circ} \\
3 \stackrel{\circ}{\circ} \\
42^{\circ}\end{array}$ & $\mid \begin{array}{r}14 \circ \\
2 \stackrel{\circ}{\circ} \\
2 \dot{\alpha} \\
18\end{array}$ & $\begin{array}{c}11 \\
0 \\
08 \\
11\end{array}$ & ᄋ $\mid \begin{array}{l}4 \\
1 \\
0 \\
5 \\
5\end{array}$ & $\mid \begin{array}{l}7 \\
0 \\
08 \\
7\end{array}$ & $\begin{array}{r}414 \\
145 \\
15 \\
674\end{array}$ \\
\hline
\end{tabular}

Epididymitis gonorrhoica.

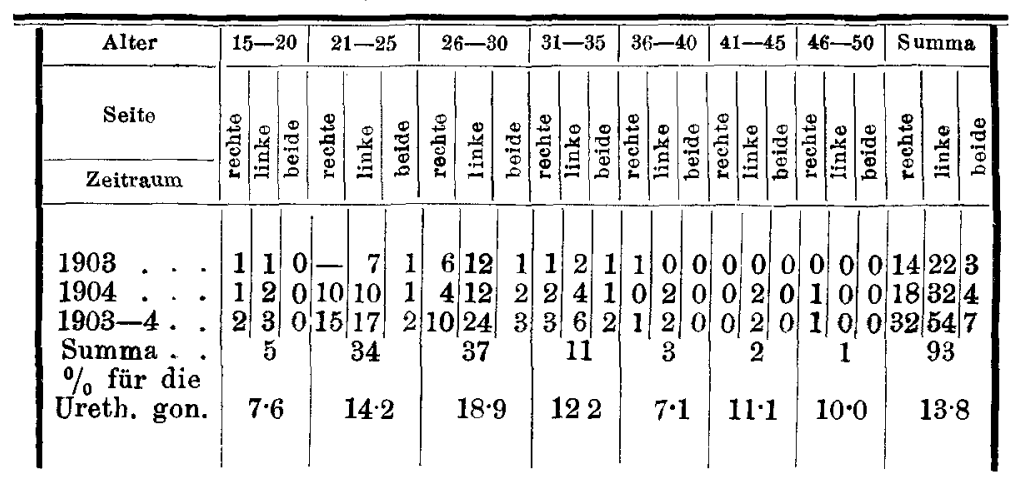


Nach meiner statistischen Untersuchung ist das Entstehen der Nebenhodenentzündung, wie es die VIII. Tabelle zeigt, beim Beruf der Sitzarbeit, wie Maler, Schneider, Tuchhändler etc., verhältnismäßig selten beim Beruf der Schnittarbeit, wie wandernden Händlern und Sachunternehmern verhältnismäßjig häufig, beim Beruf heftiger Körperbewegung, wie Bauer, Schiffsbesatzung, Handwerker etc. verhältnismäßig selten und beim Stuhlsitzer wie Beamten, Studenten, Sekretär der Bank - oder Aktiengesellschaft am häufigsten. Die Arbeiter sind einerseits meistens körperlich gesund, können kleinere Schmerzen gut ertragen und rufen nur selten den Arzt zu Hilfe, sie binden andererseits mit einem breiten Band die Hoden fest und schützen diese vor Erschütterung und äußerem Reiz; die gebildeten Leute dagegen sind meistens nervös und leicht empfindlich, selbst ein kleiner Reiz verursacht bei ihnen eine starke Entzündung.

Tabelle VIII.

\begin{tabular}{|c|c|c|c|c|c|c|c|c|c|}
\hline 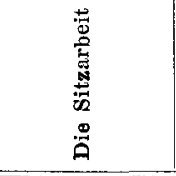 & 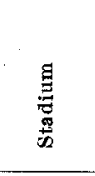 & 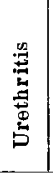 & 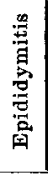 & 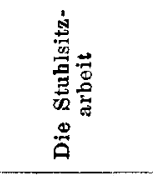 & 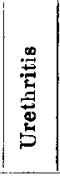 & 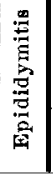 & 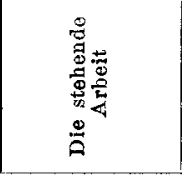 & 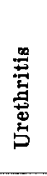 & 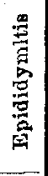 \\
\hline Maler & $\begin{array}{l}\text { chron. } \\
\text { subak. } \\
\text { akute }\end{array}$ & $\begin{array}{l}3 \\
- \\
-\end{array}$ & $\overline{-}$ & Beamte & $\begin{array}{r}23 \\
4 \\
4\end{array}$ & $\overline{5}$ & $\begin{array}{l}\text { Miso- und } \\
\text { Schoyuhändl. }\end{array}$ & $\frac{1}{1}$ & $\overline{-}$ \\
\hline Schnitzer & $\begin{array}{l}\text { chron. } \\
\text { subak. } \\
\text { akute }\end{array}$ & $\begin{array}{r}1 \\
1 \\
-\end{array}$ & $\frac{\cdots}{-}$ & Lehrer & $\begin{array}{r}6 \\
1 \\
-\end{array}$ & $=$ & Handelsm. & $\frac{1}{-}$ & E \\
\hline $\begin{array}{l}\text { Papier- } \\
\text { ankleber }\end{array}$ & $\begin{array}{l}\text { chron. } \\
\text { subak. } \\
\text { akute }\end{array}$ & $\overline{-}$ & $\begin{array}{l}- \\
- \\
-\end{array}$ & Studeut & $\begin{array}{l}99 \\
62 \\
44\end{array}$ & $\underline{23}$ & Vogelhändl. & $\begin{array}{l}1 \\
1\end{array}$ & - \\
\hline Abschreiber & $\begin{array}{l}\text { chron. } \\
\text { subak. } \\
\text { akute }\end{array}$ & $\overline{-}$ & - & Banker & $\begin{array}{r}27 \\
7 \\
4\end{array}$ & $\underline{-}$ & Apotheker & $\underline{-}$ & $\overline{-}$ \\
\hline $\begin{array}{c}\text { Haus- } \\
\text { verwalter }\end{array}$ & $\begin{array}{l}\text { chron. } \\
\text { subak. } \\
\text { akute }\end{array}$ & $\frac{1}{-}$ & - & $\begin{array}{l}\text { Geschäfts- } \\
\text { führer }\end{array}$ & $\frac{2}{-}$ & $\overline{-}$ & Brothändler & $\frac{1}{-}$ & $\overline{-}$ \\
\hline Wucherer & $\begin{array}{l}\text { chron. } \\
\text { subak. } \\
\text { akute }\end{array}$ & $\frac{6}{1}$ & - & Schreiber & $\begin{array}{l}3 \\
2 \\
1\end{array}$ & $\begin{array}{l}- \\
-- \\
-\end{array}$ & $\begin{array}{c}\text { Maschinen- } \\
\text { händler }\end{array}$ & $\overline{-}$ & - \\
\hline Fürtrag. . & & 15 & 1 & & 289 & 37 & & 10 & - \\
\hline
\end{tabular}




\begin{tabular}{|c|c|c|c|c|c|c|c|c|c|}
\hline 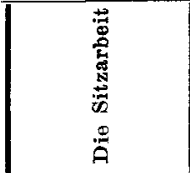 & $\begin{array}{l}\text { 基 } \\
\text { 吾 } \\
\text { in }\end{array}$ & 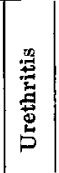 & 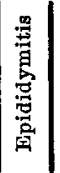 & 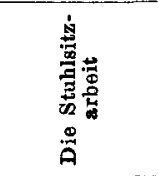 & 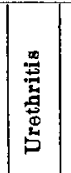 & 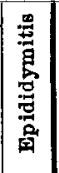 & 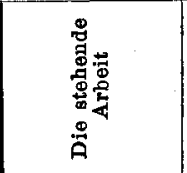 & 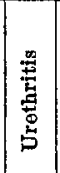 & 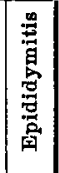 \\
\hline Übertrag . & & 15 & 1 & & 289 & 37 & & 10 & - \\
\hline Priester & $\begin{array}{l}\text { chron. } \\
\text { subak. } \\
\text { akute }\end{array}$ & $\frac{2}{2}$ & 二 & Übersetzer & $\frac{-1}{-1}$ & - & $\begin{array}{l}\text { Barett- } \\
\text { krämer }\end{array}$ & $\begin{array}{l}8 \\
1 \\
1\end{array}$ & $\underline{-}$ \\
\hline Sackhändler & $\begin{array}{l}\text { chron. } \\
\text { subak. } \\
\text { akute }\end{array}$ & $\overline{1}$ & $\frac{-}{1}$ & - & $\overline{-}$ & - & $\begin{array}{c}\text { Schuhwerk- } \\
\text { händler }\end{array}$ & $\begin{array}{l}3 \\
1 \\
1\end{array}$ & Z \\
\hline $\begin{array}{c}\text { Europäischer } \\
\text { Krämer }\end{array}$ & $\begin{array}{l}\text { chron. } \\
\text { subak. } \\
\text { akute }\end{array}$ & $\begin{array}{l}\mathbf{1} \\
\mathbf{1} \\
\mathbf{1}\end{array}$ & $\overline{-}$ & - & $\begin{array}{l}- \\
-\end{array}$ & - & $\begin{array}{l}\text { Hotel und } \\
\text { Restaurant }\end{array}$ & $\begin{array}{l}4 \\
5 \\
8\end{array}$ & $\overline{1}$ \\
\hline Buchbinder & $\begin{array}{l}\text { chron. } \\
\text { subak. } \\
\text { akute }\end{array}$ & $\overline{-}$ & - & - & - & $=$ & $\begin{array}{l}\text { alte Sachen- } \\
\text { händler }\end{array}$ & $\begin{array}{l}4 \\
1 \\
2\end{array}$ & - \\
\hline Tuchhändler & $\begin{array}{l}\text { chron. } \\
\text { subak. } \\
\text { akute }\end{array}$ & $\begin{array}{r}19 \\
4 \\
1\end{array}$ & -1 & - & $\overline{-}$ & - & $\begin{array}{c}\text { Tsukemono- } \\
\text { händler }\end{array}$ & $\frac{2}{1}$ & - \\
\hline $\begin{array}{l}\text { Kuchen- } \\
\text { steller }\end{array}$ & $\begin{array}{l}\text { chron. } \\
\text { subak. } \\
\text { akute }\end{array}$ & $\begin{array}{l}8 \\
2 \\
1\end{array}$ & - & - & - & $\overline{-}$ & Ölbändler & $\begin{array}{l}1 \\
-\end{array}$ & $\overline{-}$ \\
\hline Buchbändler & $\begin{array}{l}\text { chron. } \\
\text { subak. } \\
\text { akute }\end{array}$ & $\begin{array}{l}2 \\
1 \\
-\end{array}$ & $\frac{-}{1}$ & - & - & $\overline{-}$ & Salzhändler & $\frac{1}{-}$ & $\overline{-}$ \\
\hline Papierladen & $\begin{array}{l}\text { chron. } \\
\text { subal. } \\
\text { akute }\end{array}$ & $\begin{array}{r}1 \\
1 \\
-\end{array}$ & $=$ & - & - & - & $\begin{array}{l}\text { Bleistift- } \\
\text { fabrikant }\end{array}$ & $\frac{1}{-}$ & $\begin{array}{l}- \\
- \\
-\end{array}$ \\
\hline $\begin{array}{l}\text { Händler mit } \\
\text { getr. Fischen }\end{array}$ & $\begin{array}{l}\text { chron. } \\
\text { subak. } \\
\text { akute }\end{array}$ & $\stackrel{2}{-}$ & $=$ & - & $\overline{-}$ & $\overline{-}$ & $\begin{array}{l}\text { Faden- } \\
\text { gewerker }\end{array}$ & $\begin{array}{l}6 \\
1 \\
1\end{array}$ & $\overline{-}$ \\
\hline $\begin{array}{c}\text { Lackwaren- } \\
\text { händler }\end{array}$ & $\begin{array}{l}\text { chron. } \\
\text { subak. } \\
\text { akute }\end{array}$ & $\begin{array}{c}3 \\
- \\
-\end{array}$ & - & - & $\begin{array}{l}- \\
-\end{array}$ & - & Kellner & $\begin{array}{r}10 \\
1 \\
-\end{array} \mid$ & $\frac{-}{2}$ \\
\hline $\begin{array}{c}\text { Metallwaren- } \\
\text { händler }\end{array}$ & $\begin{array}{l}\text { chron. } \\
\text { subak. } \\
\text { akute }\end{array}$ & $\begin{array}{c}3 \\
-\end{array}$ & - & - & - & - & Buchdrucker & $\frac{2}{1}$ & 二 \\
\hline $\begin{array}{c}\text { Regen- } \\
\text { mantelhändl. }\end{array}$ & $\begin{array}{l}\text { chron. } \\
\text { subak. } \\
\text { akute }\end{array}$ & $\begin{array}{l}1 \\
-\end{array}$ & $\overline{-}$ & - & - & - & $\begin{array}{l}\text { Gemüse- } \\
\text { händler }\end{array}$ & $\begin{array}{r}2 \\
2 \\
-\end{array}$ & $\frac{-}{1}$ \\
\hline Bürstenhdl. & $\begin{array}{l}\text { chron. } \\
\text { subak. } \\
\text { akute }\end{array}$ & $\begin{array}{l}1 \\
- \\
-\end{array}$ & - & 一 & - & - & $\begin{array}{c}\text { Gebrannte } \\
\text { Imo-Händler }\end{array}$ & $\underline{-}$ & - \\
\hline Fürtrag. . & & 74 & 5 & & 290 & 37 & & 82 & 5 \\
\hline
\end{tabular}


Ein Beitrag zur Pathol. und Statistik der Epididymitis gon. 251

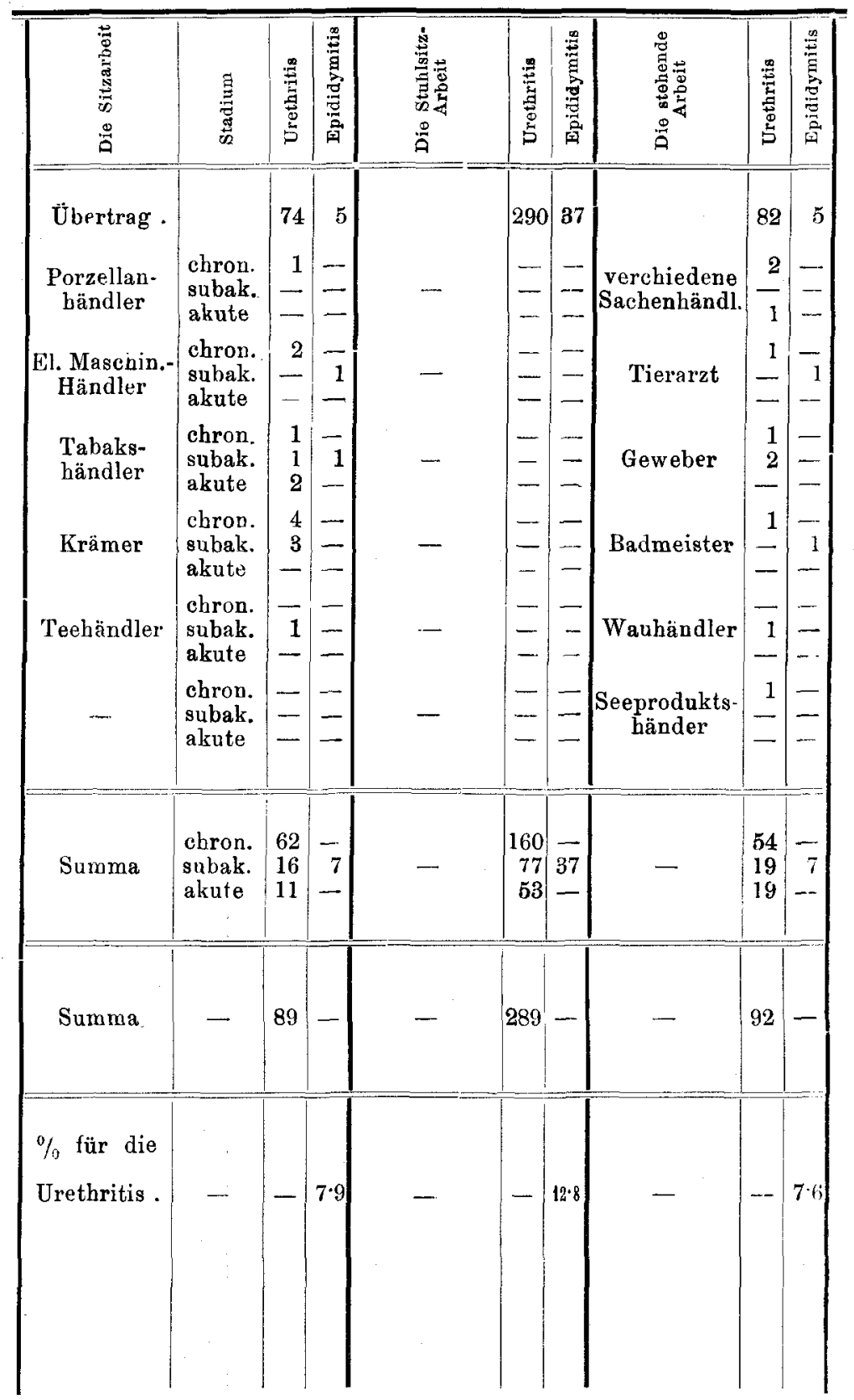




\begin{tabular}{|c|c|c|c|c|c|c|c|c|c|c|c|}
\hline 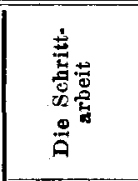 & 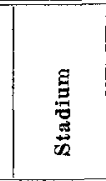 & 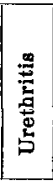 & 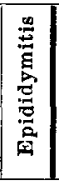 & 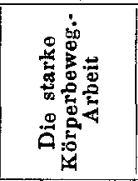 & 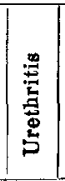 & 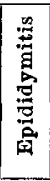 & 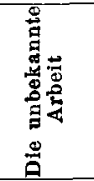 & 苞 & 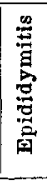 & 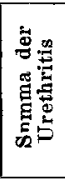 & 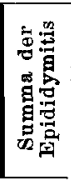 \\
\hline $\begin{array}{l}\text { Polizei- } \\
\text { diener }\end{array}$ & $\begin{array}{l}\text { chron. } \\
\text { subak. } \\
\text { akute }\end{array}$ & $\begin{array}{l}3 \\
- \\
-\end{array}$ & $\overline{-}$ & Schiffer & $\begin{array}{r}1 \\
1 \\
-\end{array}$ & 二 & $\begin{array}{l}\text { Kauf- } \\
\text { mann }\end{array}$ & $\frac{3}{-}$ & $\frac{-}{2}$ & $\overline{-}$ & $\bar{z}$ \\
\hline Postbote & $\begin{array}{l}\text { chron. } \\
\text { subak. } \\
\text { akute }\end{array}$ & $\frac{1}{1}$ & - & $\begin{array}{l}\text { Hand- } \\
\text { werker }\end{array}$ & $\begin{array}{l}\mathbf{5} \\
\mathbf{5} \\
2\end{array}$ & 1 & $\underset{\text { Bein }}{\text { Beruf }}$ & $\begin{array}{r}28 \\
4 \\
2\end{array}$ & $\frac{-}{4}$ & $\bar{z}$ & $\overline{-}$ \\
\hline $\begin{array}{l}\text { Schnitt- } \\
\text { händler }\end{array}$ & $\begin{array}{l}\text { chron. } \\
\text { subak. } \\
\text { akute }\end{array}$ & $\frac{1}{-}$ & - & Wäscher & $\begin{array}{r}1 \\
1 \\
-\end{array}$ & - & - & 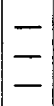 & $\begin{array}{l}- \\
-\end{array}$ & $=$ & E \\
\hline Lieferant & $\begin{array}{l}\text { chron. } \\
\text { subak. } \\
\text { akute }\end{array}$ & $\begin{array}{r}3 \\
1 \\
-\end{array}$ & $\frac{-}{1}$ & $\begin{array}{l}\text { heftiger } \\
\text { Arbeiter }\end{array}$ & $\frac{5}{5}$ & $\begin{array}{c}- \\
2 \\
-\end{array}$ & - . & $\overline{-}$ & $=$ & - & - \\
\hline Barbier & $\begin{array}{l}\text { chron. } \\
\text { subak. } \\
\text { akute }\end{array}$ & $\begin{array}{r}1 \\
2 \\
-\end{array}$ & -1 & Bauer & $\begin{array}{r}48 \\
8 \\
14\end{array}$ & $\begin{array}{c}- \\
- \\
-\end{array}$ & - & - & $\begin{array}{l}- \\
-\end{array}$ & $\bar{z}$ & $\begin{array}{l}- \\
-\end{array}$ \\
\hline $\begin{array}{l}\text { Kohlen- } \\
\text { händler }\end{array}$ & $\begin{array}{l}\text { chron. } \\
\text { subak. } \\
\text { akute }\end{array}$ & $\frac{4}{1}$ & $\frac{-}{1}$ & $\begin{array}{l}\text { Seiden- } \\
\text { würmer- } \\
\text { züchter }\end{array}$ & $\frac{-}{1}$ & 二 & - & - & $\begin{array}{l}- \\
-\end{array}$ & $\overline{-}$ & $\bar{z}$ \\
\hline Schneider & $\begin{array}{l}\text { chron. } \\
\text { subak. } \\
\text { akute }\end{array}$ & $\begin{array}{l}2 \\
1 \\
2\end{array}$ & $=$ & $\begin{array}{c}\text { Zimmer- } \\
\text { mann }\end{array}$ & $\begin{array}{l}8 \\
\mathbf{1} \\
1\end{array}$ & $\overline{-}$ & - & - & $\begin{array}{l}- \\
-\end{array}$ & $=$ & $\overline{-}$ \\
\hline $\begin{array}{c}\text { Holz- } \\
\text { händler }\end{array}$ & $\begin{array}{l}\text { chron. } \\
\text { subak. } \\
\text { akute }\end{array}$ & $\frac{1}{-}$ & $=$ & Maurer & $\frac{2}{-}$ & $\frac{-}{-}$ & - & - & $\begin{array}{l}- \\
-\end{array}$ & $\overline{-}$ & $\begin{array}{l}- \\
- \\
-\end{array}$ \\
\hline $\begin{array}{l}\text { Mörtel- } \\
\text { händler }\end{array}$ & $\begin{array}{l}\text { chron. } \\
\text { subak. } \\
\text { akute }\end{array}$ & - & $\overline{-}$ & $\begin{array}{l}\text { Tohfu- } \\
\text { werker }\end{array}$ & $\frac{1}{1}$ & $\frac{-}{1}$ & - & - & - & $\overline{-}$ & - \\
\hline $\begin{array}{l}\text { Fisch- } \\
\text { händler }\end{array}$ & $\begin{array}{l}\text { chron. } \\
\text { subak. } \\
\text { akute }\end{array}$ & $\frac{2}{-}$ & $=$ & $\begin{array}{c}\text { Reis- } \\
\text { händler }\end{array}$ & $\begin{array}{l}7 \\
2 \\
1\end{array}$ & $\frac{-}{1}$ & - & $\frac{-}{-}$ & $\frac{-}{-}$ & - & E \\
\hline $\begin{array}{l}\text { Wasser- } \\
\text { müller }\end{array}$ & $\begin{array}{l}\text { chron. } \\
\text { subak. } \\
\text { akute }\end{array}$ & $\underline{-1}$ & $=$ & Wagner & $\frac{2}{1}$ & $\frac{1}{-}$ & - & - & - & - & E \\
\hline Soldat & $\begin{array}{l}\text { chron. } \\
\text { subak. } \\
\text { akute }\end{array}$ & $\begin{array}{r}4 \\
2 \\
-\end{array}$ & $\begin{array}{l}- \\
-\end{array}$ & Schmied & $\frac{1}{-}$ & $=$ & - & $\overline{-}$ & $\begin{array}{l}- \\
- \\
-\end{array}$ & $\overline{-}$ & $\begin{array}{l}- \\
-\end{array}$ \\
\hline $\begin{array}{c}\text { Milch- } \\
\text { händler }\end{array}$ & $\begin{array}{l}\text { chron. } \\
\text { subak. } \\
\text { akute }\end{array}$ & $\mid \begin{array}{c}2 \\
-- \\
--\end{array}$ & $=$ & - & $=$ & - & - & - & $\overline{-}$ & $=$ & $=$ \\
\hline Fürtrag & & 37 & 4 & & 125 & 12 & & 37 & 6 & - & - \\
\hline
\end{tabular}


Ein Beitrag zur Pathol. und Statistik der Epididymitis gon. 253

\begin{tabular}{|c|c|c|c|c|c|c|c|c|c|c|c|}
\hline 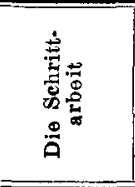 & 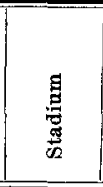 & 胥 & 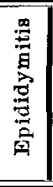 & 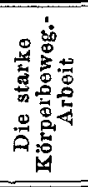 & 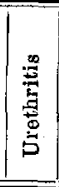 & 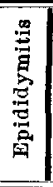 & 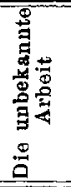 & 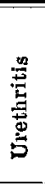 & 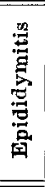 & 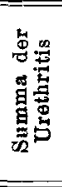 & 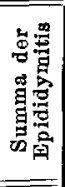 \\
\hline Übertrag & - & 37 & 4 & - & 125 & 12 & - & 37 & 6 & - & $\rightarrow$ \\
\hline Diener & $\begin{array}{l}\text { chron. } \\
\text { subak. } \\
\text { akute }\end{array}$ & $\frac{-}{1}$ & 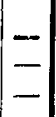 & - & - & $\begin{array}{l}-- \\
--\end{array}$ & - & - & $\overline{-}$ & - & $\overline{-}$ \\
\hline $\begin{array}{l}\text { Pflanzen- } \\
\text { händler }\end{array}$ & $\begin{array}{l}\text { chron. } \\
\text { subak. } \\
\text { akute }\end{array}$ & $\frac{1}{-}$ & 1 & - & - & - & - & - & - & - & $\overline{-}$ \\
\hline $\begin{array}{l}\text { Blumen- } \\
\text { händler }\end{array}$ & $\begin{array}{l}\text { chron. } \\
\text { subak. } \\
\text { akute }\end{array}$ & $\frac{1}{-}$ & - & - & - & - & - & - & - & ב & $=$ \\
\hline Erzeuger & $\begin{array}{l}\text { chron. } \\
\text { subak. } \\
\text { akute }\end{array}$ & $\frac{1}{-}$ & - & - & - & - & $\rightarrow$ & - & - & 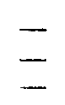 & - \\
\hline Mäkler & $\begin{array}{l}\text { chron. } \\
\text { subak. } \\
\text { akute }\end{array}$ & - & z & - & $\begin{array}{l}- \\
- \\
-\end{array}$ & $\overrightarrow{-}$ & - & - & - & - & $\overline{-}$ \\
\hline Summa. & $\begin{array}{l}\text { chron. } \\
\text { subak. } \\
\text { akute }\end{array}$ & $\begin{array}{r}27 \\
9 \\
5\end{array}$ & $-\overline{6}$ & - & $\begin{array}{l}80 \\
20 \\
25\end{array}$ & $\frac{-}{12}$ & - & $\begin{array}{r}31 \\
4 \\
2\end{array}$ & $\begin{array}{r}- \\
-\end{array}$ & $\begin{array}{l}414 \\
145 \\
115\end{array}$ & 75 \\
\hline Summa . & - & 41 & - & - & 125 & - & - & 37 & - & 674 & - \\
\hline $\begin{array}{l}\% \text { für } \\
\text { Urethri }\end{array}$ & $\begin{array}{r}\text { die } \\
\text { ritis }\end{array}$ & - & $14 \cdot 6$ & - & - & $9 \cdot 6$ & - & - & 16.5 & - & $11 \cdot 1$ \\
\hline
\end{tabular}

7. Wie die ärztliche Pflege eines Trippers die Nebenhodenentzündung herbeiführenkann!

Wenn die ärztliche Behandlung des Trippers passend ist, so ist es selbstrerständlich klar, daß die Nebenhodenentzündung größtenteils vermieden wird. Falls aber die ärztliche Behandlung sich unrichtig gestaltet, so ist die Folge davon wohl bedauernswert; die Epididymitis entsteht dann viel schneller als beim natürlichen Verlaufe. Nach Le Fort waren 
vou seinen 576 Kranken der Epididymitis gonorrhoica 264 nicht ärztlich behandelte. Unter meinen 93 Kranken haben 19 von dem Entstehen der Nebenhodenentzündung gar keine Arznei eingenommen, 47 haben nach dem Tripper bald Apothekerarznei (meistens Balsammittel), bald Arztarznei (meistens Balsammittel) genossen, aber noch keine lokale Behandlung bekommen, 27 wurden zunächst mit Urethralinjektion gepflegt, aber sind durch verschiedene Motive der Nebenhodenentzündung anheim gefallen. Daß meine Fälle verhältnismäßig wenige unbehandelte Kranke enthält, läßt sicb dadurch erklären, daß ich auch diejenigen Kranken, welche Apothekerarznei aufgenommen hatten, zu behandelten Kranken mitgezählt habe. Die Kranken, die an den Harnröhren eine ärztliche Behandlung bekamen, habe ich von den andern, die bloß innere Behandlung bekamen, genau unterschieden und gewußt, daß diese weit zahlreicher als jene sind. So kann ich den Schluß ziehen, daß oft behandelte Tripperkranke nur seltener der Nebenhodenentzündung anheimfallen im Vergleich mit den wenig behandelten Tripperkranken (s. IX. Tabelle).

\section{Veranlassende Momente.}

Hierüber sind die Meinungen von Autoren einig. Z e is s 1 (13) sah $8 \mathrm{mal}$, daß der Coitus nach einigen Tagen die Nebenhodenentzüudung erweckt. Jordan spricht sich aus, daß Coitus, Bougierung und andere instrumentale Eingriffe nicht nur dem akuten, sondern auch dem chronischen Tripper zu Schaden kommen. Meine Untersuchungen bezüglich der veranlassenden Momente sind in der IX. Tabelle zusammengestellt. Wie diese zeigt, ist die Nebenhodenentzündung erweckt: $8 \mathrm{mal}$ durch die Urethralinjektion mit 1\% Protargollösung, 9 mal durch die Injektion mit $1-2 \%$ Albarginlösung, 4 mal durch die Prostatamassage an der akuten und chronischen Prostatitis, einmal durch das Auftreten des Samens als Folge der Liebeständelei und übrigens oft durch verschiedene Veranlassungen wie Körperbewegung, zu Fuß gehen, Unmäßigkeit usw. Aber forcierte Bewegung, Coitus und Injektion ge- 
Ein Beitrag zur Pathol, und Statistik der Epididymitis gon. 255

stalten sich nicht immer als veranlassende Momente. Denn ich habe persönlich gesehen, daß zeitweise ein ruhig liegender Prostatitiskranker auch angegriffen wird. Diesem gab ich ein flüssiges Mittel von Decoctum foliae uvea ursi und Urotropin und ließ ihn täglich $2 \mathrm{mal}$ je eine Stunde eine Wärmung mittelst der Arzbergelschen Prostatasonde nehmen; noch davon wurden eine Epididymitis sinistra und Funiculitis mit einer heftigen Steigerung der Körpertemperatur erweckt und die linke Iliacalgrube verursachte heftige Schmerzen.

Tabelle IX.

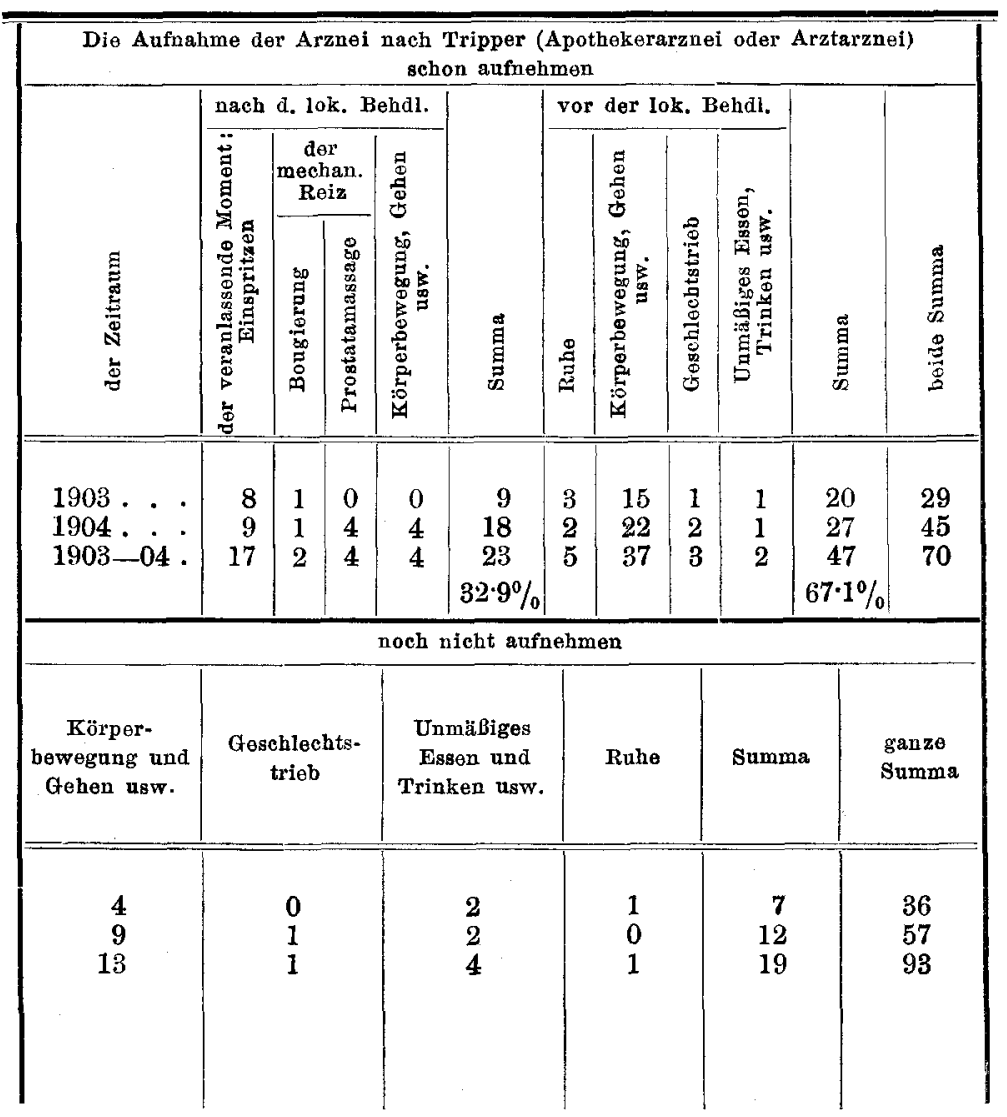


9. Das häufigere Aufkommen der Nebenhodenentzündung auf der linken Seite ist anatomisches Verhältnis.

Finger, Joseph und Gebert erkennen, daß das Aufkommen der Nebenhodenentzündung auf der linken Seite ein wenig häufiger sei als auf der anderen Seite. Zeissl, Jordan und meine Fälle zeigen, da $\beta$ es bestimmt auf der linken Seite zahlreicher ist. Die Meinung, diese letzte Tatsache sei wohl einem anatomischen Grunde zuzuschreiben, ist nicht grundlos. Ich habe bei denjenigen Tripper- und Syphiliskranken, welche noch nicht einmal an der Hode gelitten haben, die Höhe der beiden herabhängenden Hoden gemessen und die Differenzen der Höhe der beiden Seiten verglichen. Wie es die X. Tabelle zeigt, hatten 100 unter 138 Leuten an der linken Seite die länger herabhängende Hode, die Differenz beträgt durchschnittlich $0.78 \mathrm{~cm}, 32$ hatten aber an der rechten Seite länger herabhängende Hode und zwar war die rechte Hode $0.71 \mathrm{~cm}$ länger herabhängend als die linke. Die übrigen sechs hatten ebenmäßige Hoden. Wenn es also der Fall ist, daß die linke Hode gewöhnlich länger herabhängt als die rechte, so muß die linke Hode dem Druck und Zug mehr ausgesetzt sein, als die rechte. Diese äußeren Reize verursachen Bluthyperämie an der betreffenden Nebenhode und die Samenröhre erleidet zur Zeit des schlechten Stuhlganges die Blutstauung wegen der Anschwellung des s-förmigen Teils, so daß das Eindrängen der Tripperbakterien hier mehr erleichtert wird, als auf der anderen Seite. Das ist der Grund, warum ich die größere Häufigkeit auf der linken Seite für anatomisch halte. Aber der lange Herabhang der Hode an sich hat keinen nennenswerten Einfluß auf das Vorkommen der Entzündung, sondern die äußeren Reize sind hier maßgebend. Hinge z. B. auf der rechten Seite die Hode ganz kurz, so könnte doch die Entzündung ebenfalls häufig sein, wenn die äußeren Reize groß und häufig wären. So habe ich z. B. persönlich einen Kranken gesehen, dessen rechte Hode um $2 \mathrm{~cm}$ länger herabhing als die linke; ihm wurde 
Ein Beitrag zur Pathol. und Statistik der Epididymitis gon. 257

die Urethrotomia externa operiert, er lag nun ganz ruhig im Bette und doch erlitt ex eine Nebenhodenentzündung auf der linken Seite.

Der Grund war folgender: Man hob ihm bei jedem Verbandwechseln nach dem Harnlassen die linke Hode auf. Dies war der Reiz, welcher aufs neue die linke Nebenhodenentzündung verursachte. Im großen und ganzen ist die Nebenhodenentzündung auf der linken Seite häufiger.

Tabelle $\mathrm{X}$.

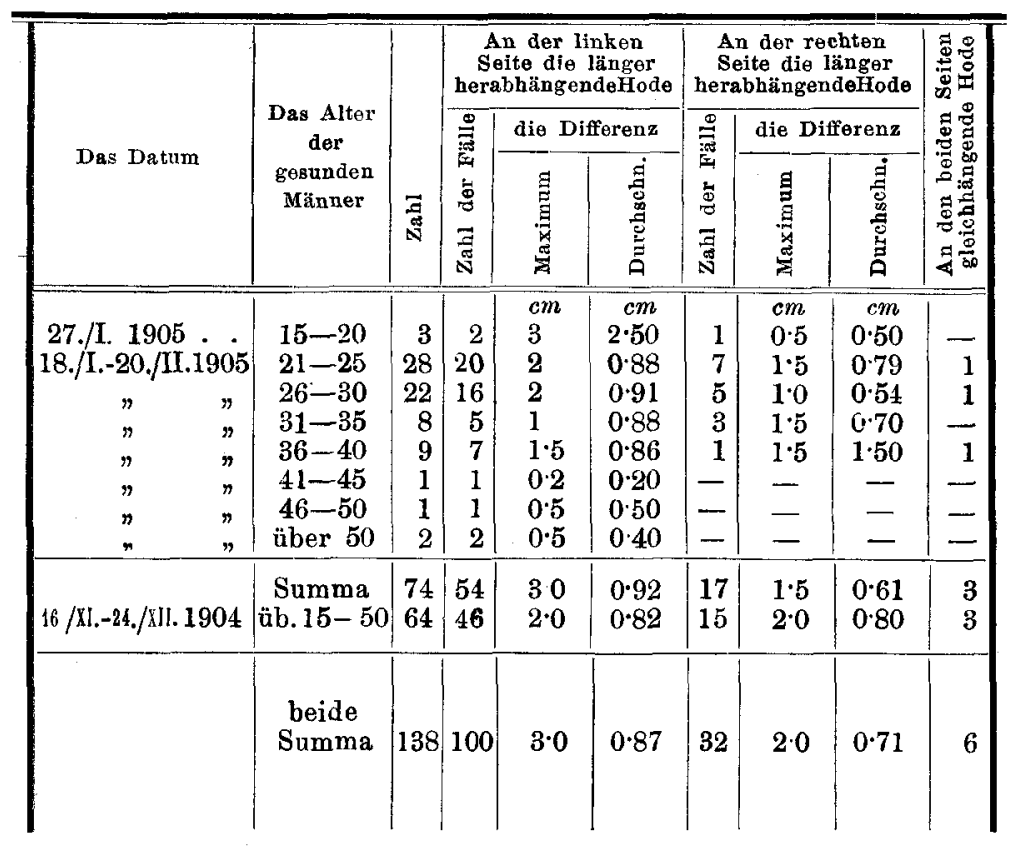

10. Ursache, warum die Entzündung der Nebenhoden meistens nicht nebeneinander, sondern hintereinander vorkommt und warum sie gewöhnlichnur auf irgend eine Seite beschränkt ist.

Wie oben gesagt, werden die äußeren Reize, wie Druck, Schlag, Zug usw., auf irgend eine Seite gerichtet und rufen in der Nebenhode und Samenleitung die Hyperämie herror Arch. f. Dermat. u. Syph. Bd. LXXXIX. 
und erleichtern so auf der betreffenden Seite die Fortpflanzung der Bakterien. Und nachdem auf einer Seite die Entzündung der Samenleitung oder Nebenhoden vorgekommmen ist, so wird der Kranke auf einmal genötigt, sich mäßig und vorsichtig zu verhalten und er schützt die Hoden kleinmütig und sorgt für die ruhige Pflege, daher wird die Entzündung der anderen Seite im voraus gehütet. Dazu noch kann man auch noch daran denken, daß wenn die eine Seite krank wird, die andere von selbst energischer wird, da diese für die $\mathbf{E r}$ krankung der anderen Seite eine kompensatorische Funktion unternehmen muß. Diese Notwendigkeit und die Vermehrung der Lebenskräfte der Gewebezellen im betreffenden Teile können auch, nach meiner Meinung, die Erkrankung der zweiten Seite vermeiden.

11. Das Verhältnis zwischen Nebenhodenentzündung und Prostatitis.

Astrus (1754) und Despres (1878) behaupteten, daß die Nebenhodenentzündung wegen der Stauung der Samenflüssigkeit, welcher als eine natürliche Folge der Urethritis gonorrhoica anzusehen ist, erweckt wird. Finger protestiert dagegen, früher hielt man die Stauung der Samenflüssigkeit für die Ursache der Nebenhodenentzündung, da diese Staung an der Spermatis und in der Hode einen zjehenden Schmerz die sogen. Spermatalgie fühlen läßt; in der Tat wird die Nebenhodenentzündung durch das Austreten der Samenflüssigkeit erweckt. Lucas (17) und Colombini (18) behaupten, daß sich die Epididymitis gonorrhoica namentlich oft mit einer Prostatitis kompliziert und bringen uns die Prozentzahl $62.5 \%$ unter ihren 64 Kranken. Lucas sah unter seinen Nebenhodenentzündungskranken stets bei der Hälfte die Prostatitis. Ich habe bei 68 unter meinen 75 Kranken die Prostata betastet: bei 22 war der Zustand der akuten Prostatitis auffallend, der Prostata stark spannte sich pflaumengroß, verursachte heftigen Schmerz bei der Fingerbetastung und dicker Eiter wurde durch die Fingermassage von der Urethralmündung 
herausgepreßt; bei den 29 wurden die beiden Portio der Prostata zeigefingergro $\beta$, begleitet von etwas derb drückenden Empfindung und schied dünnen eitrigen Schleim aus; bei den 4 wurde der Eiterausfluß vermißt; bei den 8 war die Druckempfindung ganz gering, während das Austreten des Schleims bemerkbar war; die 3 fühlten keine Druckempfindung und ließ er doch den Schleim austreten; die 2 trugen endlich die derb hypertrophierte Prostata, hatten aber weder den Druckschmerz noch Ausfluß der Flïssigkeit. Außerdem habe ich von Zeit zu Zeit die Vesiculitis spermatii in Gesellschaft mit dem Druckschmerz gesehen aber nicht in meine ernste Untersuchung gezogen. So ist es nicht zuviel gesagt, wenn ich behaupte, daß die Prostatitis die Ursache der Nebenhodenentzündung ist. Die Ductus ejaculatorius geht durch die Mitte der Prostatica hindurch und mündet an den beiden Seiten des Colliculus seminalius. Falls also die Prostatitis einmal vorkommt, so geht die Entzündung zum Ductus ejaculatorius über und bei der Zelleninfiltration in der Prostata wird der Ductus ejaculatorius sogar abgedrückt und ihre Mündung verengt, so daß in dem oberen Teil des Ductus ejaculatorius eine Blutzufuhr stattfindet und auf den Ductus ejaculatorius einen schädlichen Einfluß übt, bis endlich die Nebenhode angegriffen wird.

Fassen wir das oben gesagte hier kurz zusammen:

1. Di e Häufigkeit der Nebenhodenentzündung. Unter 674 Kranken der Urethritis genorrhoica gab es 75 Epididymitiskranke, also $11 \cdot 1 \%$. Zählten wir auch die Fälle in der Anamnese mit, so betrugen sie 93 , d. i. $13.8 \%$. Was

2. die Lokalis tion anbelangt, so betrugen die linksleidenden 54, die rechtsleidenden 32 unter den ganzen 93 Kranken, die Prozentzahl ist also links 58.1\% und rechts $34.4 \%$. 7 Kranke litten an beiden Seiten, d. i. $7 \%$.

3. Die Zeit des Auftretens ist hauptsächlich ein bis 4.5 Wochen nach dem Tripper, also die Übergangszeit rom akuten zum subakuten oder chronischen, jedenfalls die 
Zeit, wo die Urethritis posterior aufgekommen ist. 50 unter meinen 82 Fällen beweisen dies tatsächlich. Je später, wird die Häufigkeit immer mehr kleiner; aber im Exacerbationsstadium der Gonorrhoe während des chronischen Verlaufs ist die Häufigkeit verhältnismäßig groß.

4. Die Jahreszeiten. Die Prozentzahl vermehrt sich in der Änderungsperiode des Klimas, d. h. im März, Juni und November, da das Klima die Erhaltung der Gesundheit schwierig macht und namentlich im Frühling und Herbst der Mensch sinnlich wird und sich weniger beherrscht.

5. Das Alter. Im Alter, wo die akute, resp. subakute Urethritis häufig vorkommt, ist die Nebenhodenentzündung auch zahlreich. Nur Leute unter 20 Jahren sind aus Furcht vor der häßlichen Erkrankung mäßig und werden außerdem von forcierter Arbeit nicht gedrückt, da sie gewöhnlich noch keinen bestimmten Beruf haben. So ist die Prozentzahl bei ihnen wohl klein und macht eine Ausnahme.

6. De r Beruf hat keinen nennenswerten Einfluß auf die Nebenhodenentzündung. Gleichwohl ist es der Fall, daß die Sitzarbeit nur selten diese Erkrankung veranlaßt. Übrigens sind das Temperament und die körperliche Stärke maßgebend, so daß geistig tätige Leute leicht dieser Erkrankung anheim fallen, während Arbeiter mit verhältnismäßig wenigen Erkrankungsfällen fortkommen.

7. Ärztlich behandelte Kranke erleiden seltener im Vergleich mit den unbehandelten Nebenhodenentzündungen. Die veranlassenden Momente bilden meistens forcierte Bewegung, Coitus und unrichtige ärztliche Behandlung.

8. Bezüglich der gesunden Hode hatten unter allen 138 Leuten 100 Leute an der linken, 32 an der rechten längere Hoden, 6 an den beiden Seiten ebenmäßig herabhängende Hoden. So ist die Hode an der linken Seite eher dem Druck und Trauma von außen ausgesetzt, als an der rechten. Deshalb ist die größere Häufigkeit des Auftretens an der linken Seite ganz anatomisch.

9. Daß die Nebenhodenentzündung meistens auf irgend eine Seite beschränkt ist und nur selten an den beiden Seiten vorkommt, läßt sich folgendermaßen erklären: Wenn eine 
Seite angegriffen wird, so wird man vor der Erkrankung vorsichtig, benimmt sich mäßig und pflegt die Ruhe des Körpers und vermeidet auf diese Weise die Erkrankung der anderen Seite im roraus. Es ist auch sehr wahrscheinlich, daß die Erkrankung der einen Seite die kompensatorische Funktion der anderen Seite veranlaßt und somit die letzte mit der neuen Lebenskraft der Gewebezellen versehen wird, um gegen die äußeren Reize immer mehr standhafter werden zu können.

10. Es gibt kaum einen Kranken mit der Nebenhodenentzündung, der nicht mehr oder weniger an Prostatitis leidet, da einerseits die Entzündung dorthin übergeht und andererseits in der Prostata eine Zelleninfiltration erweckt, die Ductus ejaculatorius abgedrückt wird, so daß an der Kanalwand ihres oberen Teils eine Hyperämie stattfindet und die Fortpflanzung der Gonokokken erleichtert.

Meinem hochverehrten Lehrer, Herrn Professor Dr. K. Dohi, spreche ich hier für die liebenswürdige Leitung und Unterstützung meinen wärmsten Dank aus. 


\section{Literatur.}

1. Lesser. Encyklopädie der Haut- und Geschlechtskrankheiten. Leipzig 1900. p. 122.

2. F in g e r. Die Blennorrhoe der Sexualorgane. L eipzig 1901. p. 293.

3. Brockhart. Über die pseudo-gonorrhoische Entzündung der Harnröhre und des Nebenhodens. Monatsh. f. pr. D. 1886. Bd. V. p. 134.

4. Gustav. Über die Pathogenese der blennorrhoischen Epididymitis und über Versuche, dieselben durch Punktion zu behandeln. Deutsche med. Wochenschrift. 1903. Nr. 40.

5. Bernstein. A case of infection of the epidilymis and Tunica vaginalis by Friedländers bacillus. Amer. journ. of the med. sciences 1903 . Ref. Zentralbl. f. d. Kr. der Harn- u. Sexualorgane v. Sedlmayr. p. 611. 6. Ga lew sky. Über chron. nicht gonorrb. Urethritis. Zentralbl. f. d. Kr. d. Harn- u. Sexual-Organe. 1903. Bd. XIV. H. 9.

7. Karl Ritter von Hofmann. Bakterienbefunde bei ehronischer Gonorrhoe. Zentralbi. f. d. Kr. d. Harn- u. Sexualorg. 1903. Bd. XV.H. 11. 8. Els enberg. Gibt es eine idiopathische Nebenhoden- u. Hodenentzündung. Wiener med. Presse. 1893. Nr. 31 u. 32.

9. Jordan. Ein Beitrag zur Statistik d. Epididymitis gonorrhorca. Arch. f. Dermat. u. Syph. Bd. LXXII. H. 1. 1904.

10. Simonis, J. Zit. in M. Josephs Lebrbuch der Haut- und Geschlechtskrankh. 1901. Bd. II. p. 385.

11. Naught, Mc. Epididymitis from Complication of the Typhus. Brit. med. Journ. 15. Nov. 1902.

12. Gas mann. Beitr. zur Kenntnis der Gonorrhoe des Mannes, insbesondere der Prostatitis und Epidymitis. Zentralbl. f. d. Krankh. der Harn- und Sexualorgane. Bd. XV. H. 7.

13. v. Z e is s l. Lehrbuch der vener. Krankh. 1902. p. 107.

14. F in g e r. Schmidts Jahrb. 1882. Bd. CXCIII. p. 202. Ref. in (9).

15. Ok a mura, T. Die Verbreitung der venerischen Krankheiten. Japan. Zeitschr, f. Derm. u. Urologie. Bd. II. p. 49.

16. E r b. Syphilis und Tabes. Berl. klin. Woch. 1904. p. 41.

17. L u cas, G. Résultats de toucher rectal dans 285 cas d'épididy-

mitis blennorrhagiques. Ann. de derm. et de syphil. 1894. p. 1157 in (9).

18. Colom bini. Über die Häufigkeit der Prostatitis, Vesiculitis etc.

Ref. Monatsschr. f. prakt. Dermat. 1896. Bd. XXIII. p. 49. 